\title{
Electrical Fiber Optical Sensor for High-Voltage Applications
}

\author{
Markus Richter, M. Eng. \\ Dr.-Ing. Alexander Kölpin \\ Dr. Thomas Bosselman \\ Prof. Dr.-Ing. Dr.-Ing. habil. Robert Weigel \\ Markus Richter \\ Siemens AG \\ Corporate Technology \\ Research \& Technology Center \\ Günther-Scharowsky-Str. 1 \\ 91058 Erlangen, Deutschland \\ markusrichter.ext@siemens.com
}

\begin{abstract}
Controlling the energy transfer in high voltage grids implies using sophisticated instrumentation, which has to ensure effective insulation for $150 \mathrm{kV}$ to $800 \mathrm{kV}$. Several solutions were previously proposed to facilitate power supply for instrumentation operating at this potential. Most of them exploit the energy dissipated by alternating current grids, which is not possible for the recently emerging direct current grids. There, no alternating electric or alternating magnetic fields are available such that power supply by fiber optical power transmission is a commonly chosen solution. However, most of these systems involve high power laser diodes that lead to a considerable amount of problems and risks. In this paper, an electrical circuit design is proposed that reduces the amount of required energy such that alternative optical power sources can be used. It has been shown that an electrical circuit can measure a temperature and transmit the results, while it is supplied with less than $180 \mu \mathrm{W}$ of power through the fiber used for data transmission. The energy necessary for measurement and transmission of a single temperature value could be reduced to $15 \mu \mathrm{J}$. A combination of selected components, energy saving optimized design and programming was used to cut down the energy demands such that light emitting diodes coupled to a standard multi mode fiber are sufficient for power supply.
\end{abstract}

Key words: Instrumentation, Fiber Optical Power Transmission, Energy Harvesting, Low Power, Light Emitting Diodes

\section{Problem and Motivation}

The current situation of sensory for harsh environment is, that they are expensive and difficult to use. Mainly on high voltage applications the serious obstacle is the insulating between ground an high voltage potential. Thus at a lot of use cases it will be renounced or replaced by a sensor in some distance. The target is to develop some easy to use, easy to replicate, and low cost sensor systems. In the world of energy transfer grids, the biggest problem for sensory it the connection form ground potential to the high voltage potential. A commonly chosen solution is connection by glass fiber[1].
Glass is a natural insulator and it is an easy to handle connection. It is also technically possible to distribute energy through the fiber and supply the sensor. Nevertheless, it is difficult to transport a lot of energy through the fiber so it is necessary to reduce power consumption of the sensor to a minimum $[2,3]$.

\section{Solution}

To solve the problems in application, it was necessary to combine several optimized components. Starting with a high efficiency ultra low voltage power converter that has to pump the voltage of a single solar cell to the storage voltage [4]. The 
energy management has to signal that enough energy is stored and a measuring can be started, see Fig(1). A common way to do the measuring is to use a low power microcontroller and an analog or a digital sensor. The microcontroller has to schedule the measuring, data conversion, and transmitting process. A further important energy saving key component is the optimized program code to the application. It has to use the energy saving functions of the microcontroller during data conversion time of the sensor and all other delay times.

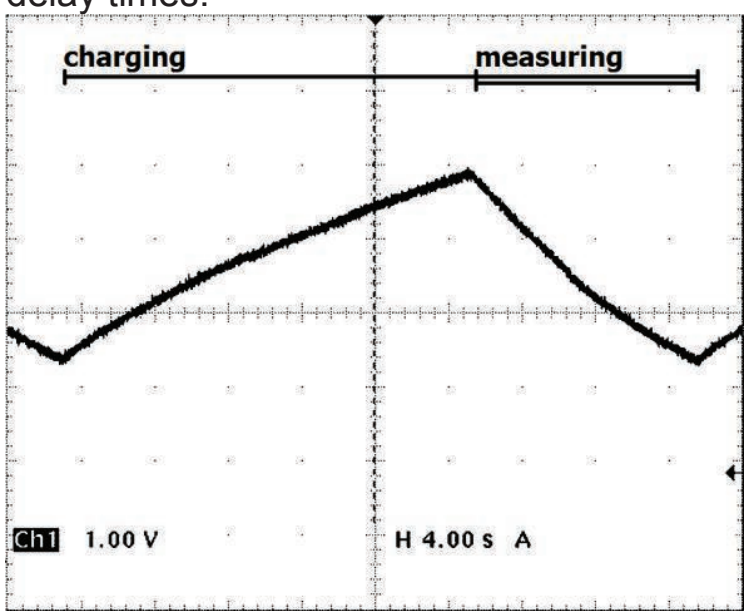

Fig. 1: Voltage of the energy storage on the sensor in paused- measuring mode

Finally, the microcontroller has to organize the data transmission. The requirement at this point is also to use as less as possible energy to transmit a high precision value. The mostly convincing method is to send a short pulse with modulated code, see in Fig(2). The first high pulse define the timebase from rising edge to the falling edge. The low time between the first and the second pulse represents the measured value and the second high time repeats the timebase. To decode the signal, a time synced decoder is not required. It is only necessary to divide negative pulse with though the positive pulse with. Another advantage of a time encoded signal is that it is possible to decode it from very weak signals. The complete signal and power is depicted in Fig(3). The key advantages of this technology are that it is possible to connect a active sensor without a separate power source and any electrical connection. Moreover, the reliability of wire connected sensors is achieved in combination with the advantages of wireless sensors like ultra high insulating, insusceptible to electrical, and magnetic fields.

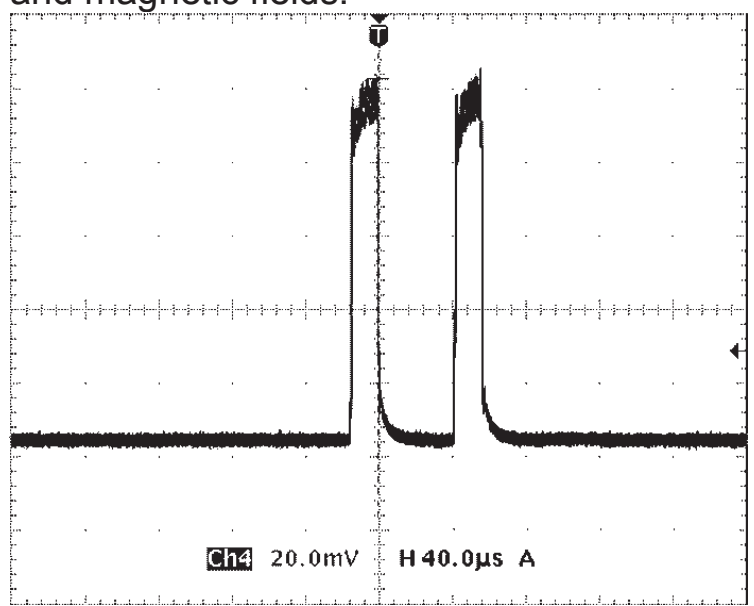

Fig. 2: Shape of received from sensor at ground receiver station 


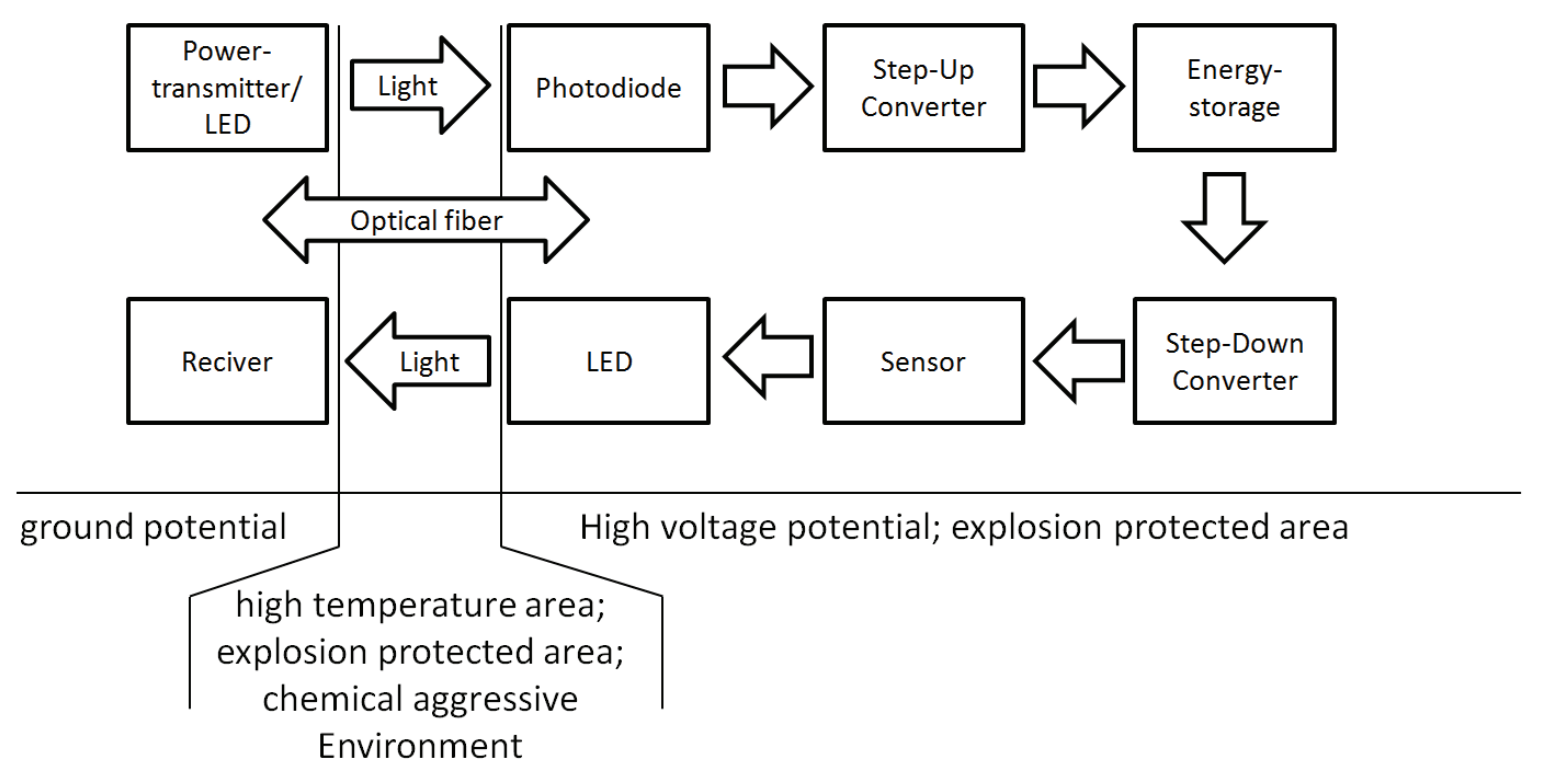

Fig. 3: Structure of power over fiber energy harvesting sensor

\section{Results}

For the development and demonstration of the sensor system, a temperature sensor was implemented in hardware. After optimization of hardware and software components, a hybrid optical sensor has been developed. It can be powered by light with less energy than a LED is able to transmit in a given time span. The total amount of energy that is necessary to execute a single measurement is $13,5 \mu \mathrm{J}$. Hence, it is possible to perform two measurements per second supplied with the power of a LED (approx 150 $\mathrm{WW}$ at $850 \mathrm{~nm}$ ) in a standard $62.5 \mu \mathrm{m}$ glass fiber.

\section{References}

[1] K. Dembowski, Energy Harvesting fuer dei Mikroelektronik, pp.24 ff, 1st edition, VDE Verlag, Berlin, 2011.

[2] F. V. B. de Nazare and M. M. Werneck, "Temperature and current monitoring system for transmission lines using power-over-fiber technology," in Instrumentation and Measurement Technology Conference (I2MTC), 2010 IEEE, 2010, pp. 779-784.

[3] Perhirin S., Audo F., Guegan M., Quintard V., Perennou A., Ghisa L., De Blasi S., Auffret Y., "A power-over-fiber system and its low consumption remote equipment for submarine applications", in OCEANS - Bergen, 2013 MTS/IEEE, 2013, 10.1109/OCEANS-Bergen.2013.6608175

[4] Texas Instruments. (2014, Dec.) BQ25504. [Online]. Available: www.ti.com/products/bq25504 